\title{
Efficient Ciphertext Policy Attribute-Based Encryption through Outsourcing with Checkability for the Internet of Things
}

\author{
Chih-Hung Wang ${ }^{1}$, Ying-Jung $\mathrm{Hsu}^{2}$ \\ ${ }^{1,2}$ Computer Science and Information Engineering, National Chiayi University
}

\begin{abstract}
Internet of Things (IoT) has been more and more popular in the recent years, this paper presents a novel approach to outsourcing the computation of the encryption with checkability in the ciphertext policy attribute-based encryption (CP-ABE) structure for the IoT. Because of the computation of the CP-ABE, the resource-constrained device in the IoT may be difficult to complete the computation. Through the way of splitting secret and sending the pairing computation to the assistant nodes, the resource-constrained device can check the returning results by the assistant nodes and generate the ciphertext with only several multiply operations. In the proposed scheme, the resource-constrained device can correctly complete the encryption through outsourcing without releasing any secrets.
\end{abstract}

Keywords: ciphertext policy attribute-based encryption, Internet of Things, encryption outsourcing, resourceconstrained device.

\section{Introduction}

In the recent years, there are lots of paper about $\mathrm{ABE}$ (attribute-based encryption). ABE, a novel scheme about access control, can use the attributes of the user to encrypt or decrypt message. It is divided into two categories: $\mathrm{CP}-\mathrm{ABE}[2]$ and $\mathrm{KP}-\mathrm{ABE}[3]$. The difference between the two methods is that the CP-ABE uses the access structure to encrypt message and the attribute set to be the key generation; the KP-ABE uses the attribute set to encrypt message and the access structure to be the key generation.

Considering the cost of the $\mathrm{ABE}$ computation, lots of issues about outsourcing or cooperation were proposed. The methods assist the resource-constrained device to run encryption or decryption under ABE scheme. In 2011, Waters et al. [7] present a CPA-secure CP-ABE outsourcing scheme. In 2014, Asim et al. [1] presented a complete method to outsource data; the proxy helps the user to handle data without pairing computation and helps the low-end mobile device running the encryption and decryption. Touati et al. [6] further developed a method of cooperative $\mathrm{CP}-\mathrm{ABE}$ (C-CP-ABE) that can help the nodes in the IoT to be able to run encryption of the CP-ABE.

In addition to helping nodes to run encryption in the IoT environment, this paper also provide the property that the nodes can check the correctness of the outsourcing data. Apart from that, the proposed scheme combine the decryption outsourcing of the $\mathrm{CP}-\mathrm{ABE}$. For instance, imaging there are lots of embedded devices for monitoring the patient's physiological situation in the hospital all day long and the monitoring data may include the patient's privacy. Therefore, the embedded devices encrypt the data and transmit the ciphertext to the medical system, and then stores the data in the system database. The doctor who has been authorized can legally view the patient physiological situation through the doctor's computer or the personal cell phone.

\section{Preliminary}

Before explaining how the proposed method works, this section simply introduces some foundation background knowledge about cryptographic techniques and defines the notations used in this paper.

\subsection{Bilinear Maps}

Let $e$ be a bilinear map $e: G \times G \rightarrow G_{T}$, where $G$ and $G_{T}$ are two multiplicative cyclic groups of prime order $p$, and $g$ is a generator. The bilinear map $e$ has the following properties: 
- Bilinearity: for all $u, v \in G$ and $a, b \in Z_{p}, e\left(u^{a}, v^{b}\right)=e(u, v)^{a-b}$.

- Nom-degeneracy: $e(g, g) \neq 1$.

We define that $G$ is a bilinear group if the group operation in $G$ and the bilinear map e:G $\times G \rightarrow G \_T$ both compute efficiently.

\subsection{CP-ABE Model}

In the CP-ABE [2] model, the message is encrypted according to the access structure and the private key is identified with a set $S$ of descriptive attributes. The message is allowed to be encrypted when it satisfy the access tree $A T$. The non-leaf node is the threshold gate and the leaf node is the attribute in the $A T$. Talking about $A T$, we have to mention the following functions:

Parent $(x)$ : return the parent of the node $x$ in the access tree $A T$.

$\boldsymbol{A t t}(\boldsymbol{x})$ : return the attributes associate with the node $x$ in the access tree $A T$.

$\operatorname{Index}(x)$ : return the order of the node $x$ in the access tree $A T$.

\subsection{Outsourcing the decryption of $\mathrm{CP}-\mathrm{ABE}$}

Outsourcing the decryption of CP-ABE model [4] mainly has five algorithms. In the following, we describe how the algorithms work respectively in detail:

- $\operatorname{Setup}(\lambda, U)$ : It takes as input the security parameter $\lambda$ and the universe description $U$. Outputs the master secrect key $M S K$ and the public key $P K$.

- $\operatorname{Encrypt}(\boldsymbol{P K}, \boldsymbol{M}, \boldsymbol{T})$ : Inputs the public key $P K$, the message $M$ and a LSSS access structure $T$. Outputs the ciphertext $C T$ with the description $T$.

- $\operatorname{KeyGen}(M S K, S)$ : Inputs $M S K$ and a set of attributes $S$. Outputs the transformation key $T K$ and the private key $S K$.

- Transform(TK,CT): Takes as input the transformation key $T K$ for a set of attributes $S$ and the ciphertext $C T$ for the LSSS access structure $T$. If $S$ does not satisfy $T$, aborting algorithm; otherwise, outputs the partially decryption ciphertext $C T$ '.

- $\operatorname{Decrypt}(\mathbf{S K}, \boldsymbol{C T})$ : Inputs the private key $S K$ and the ciphertext $C T$. If $C T$ is not the partially decryption ciphertext format, runs the Transform algorithm first. Otherwise, outputs the plaintext $P T$.

Note that the key of this method is outsourcing the ciphertext. First, the ciphertext is transferred to the El Gamal format one, and then the user can run the Decrypt algorithm by computing simple multiplications and get the plaintext $P T$.

\subsection{Batch exponentiations outsourcing with fixed base-variable}

In this part, assume that the device $D$ wants to outsource the exponentiation computation to the remote server RS, the followings would explain how to work:

- Initialize: $D$ wants to compute $g^{x_{1}}, g^{x_{2}}, \ldots, g^{x_{i}}$. In the first, $D$ generates $n$ random numbers $r_{1}, \ldots, r_{n}$, where $m$ of which could be summed to $x_{j}$, where $m$ is set close to $(n-i) / 2$ generally, $j \in[1, i]$. Second, $D$ sets $c_{k}=x_{k}-\sum_{j=1}^{m-1}$ base $_{j}$ that guaranteed there must be the sum to be $x_{k}$, where $k \in[1, i]$, base $_{j} \in Z_{p} j \in[1, m]$. Finally, $D$ generates a new set $S=\left(s_{1}, \ldots, s_{i+m+n}\right)$ with the random order, which composed of $r_{j}, c_{k}$, base $_{l}$, where $j \in[1, n], k \in[1, i], l \in[1, m]$; and a list $L$ to record the result.

- Send: $D$ sends $S$ and $g$ to the independent remote servers $R S_{1}$ and $R S_{2}$.

- Response: $R S_{1}$ and $R S_{2}$ return the corresponding result $\left(g^{S_{1}}, \ldots, g^{S_{i+m+n}}\right)$ one by one to $D$, and $D$ would compare the result. If the comparison result is different, it means at least one of them is dishonest.

- Compute: If $R S_{1}$ or $R S_{2}$ is dishonest, aborting protocol. Otherwise, for $j \in[1, i+m+n]$;

$$
S_{\text {base }}=S_{\text {base }} \cdot s_{j}, \quad S_{k}=S_{k} \quad \text { if } s_{j} \in \text { base }_{l}
$$




$$
\begin{array}{llrl}
S_{\text {base }}=S_{\text {base }}, & S_{k}=S_{k} \cdot s_{j} & \text { if } s_{j} \in c_{k} \\
S_{\text {base }}=S_{\text {base }}, & S_{k}=S_{k} & \text { otherwise }
\end{array}
$$

At the end of the Compute, $D$ completes the computation: $g^{x_{j}}=S_{\text {base }} \cdot S_{j}$, where $j \in[1, i]$.

\subsection{Notations}

The notations of the following table are used in this paper and their descriptions are explained.

\begin{tabular}{|c|c|c|c|}
\hline Notation & Description & Notation & Description \\
\hline$D$ & Device & $P K$ & Public key \\
\hline $\mathbb{P}_{\mathrm{i}}$ & Assistant node $i$ & $C T$ & Ciphertext \\
\hline$R S$. & Remote server & $P T$ & Plaintext \\
\hline$(M, \rho)$ & LSSS access structure & $r_{1 x \ldots x} r_{l}$ & Random number \\
\hline$M$ & A $l * n$ matrix & $y_{2} \cdots y_{b}$ & Random number \\
\hline$\rho$ & A function associated with the rows of $M$ to attribute & $M_{i}$ & The vector relative to the $i$-th row of the Matrix $M$ \\
\hline$S$ & Encryption exponent & $Q_{i}$ & The subset of $\lambda_{\mathrm{i}}$, where $i=1$ to $l$ \\
\hline$F O$ & a hash function $\operatorname{map}\{0,1\}^{*}$ to $G^{\square}$ & $R_{\tilde{i}}$ & The subset of $r_{i}$, where $i=1$ to $l$ \\
\hline OutPack & The subset of set $\mathrm{S}, Q_{i}, R_{\hat{i}}$, where $i=1$ to $l$ & $\mathrm{~S}$ & The subset of $\mathrm{s}$ \\
\hline$L_{i}$ & \multicolumn{3}{|c|}{ A list of the orders of data shares to $\mathrm{P}_{i}$ related to the computing operation and indicating which of them are random numbers } \\
\hline
\end{tabular}

TABLE I: Notation Table

\section{Proposed Solution of Checkability}

To run the CP-ABE scheme in the IoT environment, we have to outsource the costly computations to support the node execution successfully. The main idea of this paper is outsourcing all the exponentiation computations in the node encryption phase to the assistant nodes, such that the node can just compute the multiplications to complete the encryption algorithm.

\subsection{Network Model}

In the encryption phase, the IoT environment has lots of devices connected to each other with such as wireless sensor networks (WSNs), the embedded computing nodes are expected to be a resource-constrained devices, the unconstrained devices can cooperatively complete the computations, and a server is needed to storage and analyze the data which these embedded devices monitor. In the following, we classify the nodes into three classes [6]:

- Resource-constrained devices: the device has the ability of weak computations and low storages (such as the sensor, embedded computing device.) The resource-constrained devices are assumed to be difficult to implement the CP-ABE pairing computation, and never deliver sensitive data.

- Unconstrained devices: the device has stronger computation and storage ability than the resourceconstrained devices, and it is also called "assistant node". The unconstrained devices is assumed to be dishonest (may return the error computation result to save the resource or other target.)

- Remote server: the device has the strong computation ability and large amount storage, and it can cooperatively compute the encryption algorithm and storage ciphertext without learning any sensitive data.

In the decryption phase, the user terminal requests the ciphertext from the remote server and there is a proxy to assist the decryption without release any sensitive data.

\subsection{Assumption}

The assumptions in the paper are listed below.

- There are at least two unconstrained devices to be the neighborhood of any resource-constrained devices.

- It always shares the pairwise keys between device A and device B when A want to send message to B. The keys have to be generated before, like specific bootstrapping phase or initialization phase. 
- It is assumed that the remote server is trustworthy and is responsible for handling the outsourcing encryption and checking the correctness of the data from the assistant nodes.

\subsection{How it works}

The encryption algorithm is performed by the resource-constrain device $D$, and the inputs are the public key $P K$, plaintext $P T$ and the LSSS access structure $(M, \rho)$, where $M$ is a $l \times n$ matrix and $\rho$ is a function associate with the rows of $M$ to attribute. Then device D outsources the computation to $N$ assistant nodes $P_{a}$ (where $a=1 \sim N$ ), then output the ciphertext $C T$ along with a description of LSSS access structure $(M, \rho)$ to the remote server $R S$.

We modify the encryption phase from the method of CP-ABE [4], the other phase algorithms (Setup, KeyGen, Transform and Decrypt) are the same as the original ones in the CP-ABE (see Fig 1). In the following, we divide the encryption algorithm into several sub-phases to describe algorithm in detail:

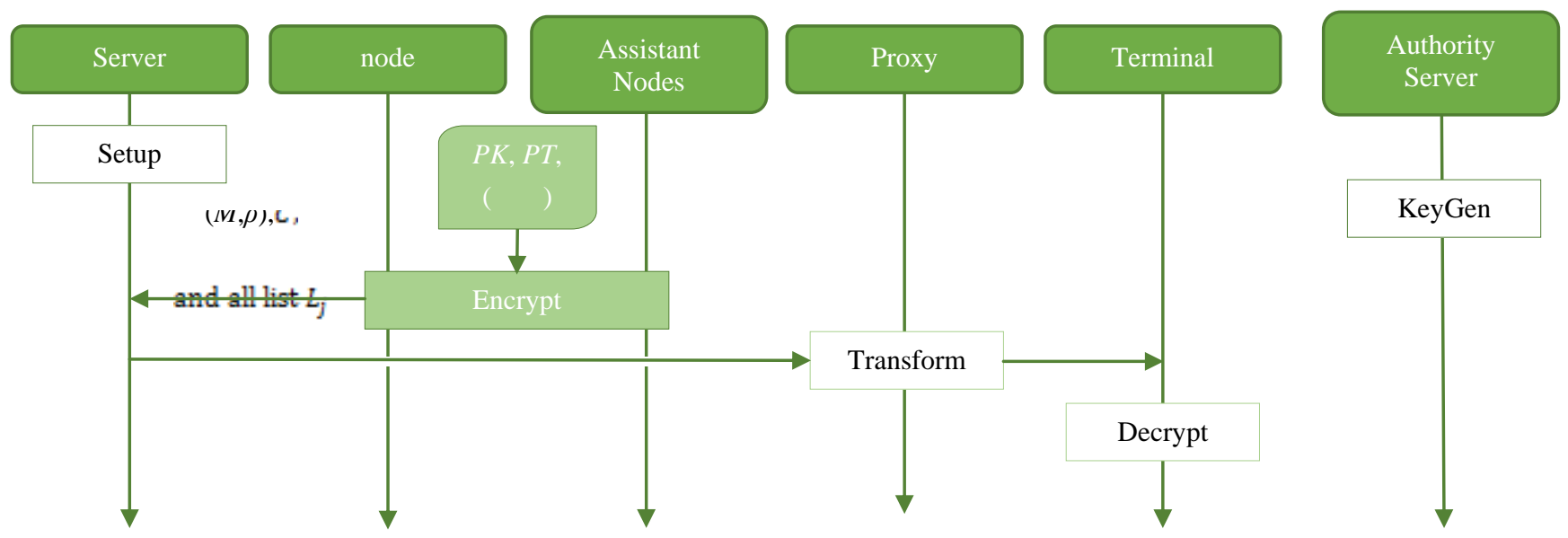

Fig 1: The outsourcing process in IoT environment

- Phase 1: The resource constrained device D has to encrypt the Plaintext PT. Device D chooses $m$ neighbours (where $m$ is bigger than 2) unconstrained devices to be the assistant nodes to outsource some exponentiation operations in the encrypt phase.

- Phase 2: The device $D$ defines LSSS access structure $(M, \rho)$ according to the attribute of the $P T$. It randomly chooses the numbers $s, y_{2}, \ldots, y_{b} \in Z_{p}^{b}$ to be a new vector $\vec{v}$ (where $s$ is the encryption exponent, and these values will be used for the encryption) and the random number $r_{1}, r_{2}, \ldots, r_{l} \in Z_{p}$. The device $D$ calculates $\lambda_{i}=\vec{v} \cdot M_{i}$, where $1 \leqq i \leqq l$ and $M_{i}$ is the vector related to the $i$-th row of the Matrix $M$.

- Phase 3: The device $D$ splits $s$ by the algorithm Split to generate the set $S=\left\{s_{1}, s_{2}, \ldots, s_{x}\right\}$ and a function $L_{0}$ where $\Sigma_{L_{0}\left(s_{i}\right) \geqq_{0} s_{i}}=s ;$ Split $\lambda_{i}, r_{i}$ for $i=1$ to $l$, to generate the set $Q_{i}, R_{i}$ and list $L_{i}$ to record the order. It sets the security degree as $T$ by the user and picks out subset from the sets $\mathrm{S}, Q_{i}, R_{i}$ into $N$ parts: $\left\{\right.$ OutPack $_{1, \ldots}$, OutPack $\left._{N}\right\}$.

- Phase 4: For each assistant node $\mathrm{P}_{a}$, the device $D$ sends the OutPack $k_{a}$ and the $\mathrm{F}(\rho(j))$ where $j=1 \sim l$, through the secure channel that means these data are encrypted by the share key $\mathrm{K}_{D P_{a}}$.

- Phase 5: Each assistant node $\mathrm{P}_{a}$ calculates the $e(g, g)^{\alpha s_{i}}, g^{s_{i}}$ and the $g^{\alpha \lambda_{j}}, g^{r_{j}}, F(\rho(j))^{-r_{j}}$ where $s_{\mathrm{i}} \in O u t P a c k_{\mathrm{a}}, j=1$ to $l$, and $F O$ is a hash function: $\{0,1\}^{*}$ to $G^{a}$; then returns the result of the $e(g, g)^{\alpha s_{i}}$ where $s_{i} \in O u t P a c k_{a}$ to $D$ by the secure channel encrypted by the share key $\mathrm{K}_{D P_{i}}$. $P_{a}$ sends the remaining result to the remote server $R S$.

- Phase 6: After receiving the result from the assistant nodes, the device $D$ running CheckResult algorithm with the list. If the algorithm returns error, $D$ stops execution. Otherwise, it computes $\widetilde{C}=P T$ 
$\prod_{L_{0}\left(s_{i}\right) \geqq_{0}} e(g, g)^{\alpha s_{i}}$, and sends $\left(M_{,} \rho\right)_{z}, \tilde{C}_{\text {, }}$ and all list $L_{j}$ to the remote server RS by the encrypted channel.

- Phase 7: After receiving all the results from the assistant nodes and the device $D$, the remote server $R S$ runs CheckResult algorithm with the list from the device $D$. If the algorithm returns error, $R S$ outputs the error message and break the algorithm; otherwise, $R S$ computes the ciphertext $C T$ :

$\tilde{C}_{,} C=g^{s}=\prod_{L_{0}\left(s_{i}\right) \geqslant 0} g^{s_{i}}{ }_{s}$ for $i=1$ to $l_{s}\left(C_{i}=g^{\alpha \lambda_{i}} * F(\rho(i))^{-r_{i}}{ }_{2} D_{i}=g^{r_{i}}\right)$.

RS stores the ciphertext as the $C T$ and the access structure $(M, \rho)$.

\section{ALGORITHM I: Split}

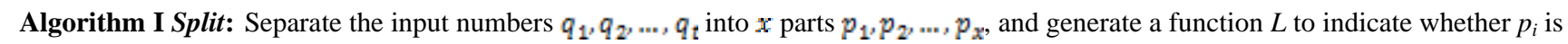
a random number.

Input: The set $H=\left(q_{1}, q_{2}, \ldots x, q_{t}\right)$ and the number $x$ to separate the set $H$ into a new set $S$ with $x$ elements.

Output: A set of number $S$ and the function $L$.

1: Generate two set of random numbers rand, base $_{j}$, where $j \in\left[1_{v}(x-t) / 2\right]$.

2: Generate $c_{k}=q_{k}-\sum_{j=1}^{(x-t) / 2}$ base $_{j}, k \in\left[1_{x} t\right]$.

3: Combine rand, base ${ }_{j}, c_{k}$ where $j \in\left[1_{x}(x-t) / 2\right]_{x} k \in\left[1_{s} t\right]_{x}$ Generate a set $S^{\prime}$ :

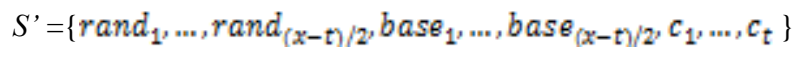

4: Shuffle the set $S$ ' to be a new set $S$ and generate a function $L$ :

$L\left(p_{i}\right)>0$, if $p_{i} \in\left\{\varepsilon_{1}{ }_{0 \times x} c_{t}\right\}$; the value of $L\left(p_{i}\right)$ represents the sequence number of $p_{i}$ in this set

$L\left(p_{i}\right)=0$, if $p_{i} \in\left\{\right.$ base $_{1, \ldots,}$ base $\left.e_{(x-t) / 2}\right\}$

$L\left(p_{i}\right)<0$, if $p_{i} \in\left\{\right.$ rand $\left._{1, \ldots x} \operatorname{rand}_{\left[x-t_{1} / 2\right.}\right\}$

5: $\quad$ Return the set $S$ and the function $L$.

\section{ALGORITHM II: PickOut}

Algorithm II PickOut: Divide set $S$ into $p$ subset; the security degree T output $Q_{1^{v}} Q_{2^{x} \text { s } x} Q_{u}$

Input: Two values, the set $S$ and the number $p$ which is going to separate set $S$ into $p$ subset.( $p$ is the number of assistant nodes)

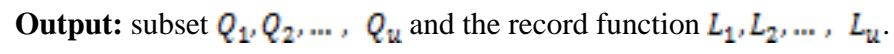

1: Set $|\mathrm{S}|$ is the number of the element of the set $S, w=\frac{|| s \mid}{p}$.

2: Set the number of the subset element $\# s u b=\frac{|s| \otimes T}{p}$

3: //Generate subset $Q_{\tilde{u}}$ :

4: for templ= 1 to $u$ do

5: //ensure that all answers would be calculated more than $T$ times and no two different assistant nodes would compute the same subset.

6: $\quad$ for $t e m p 2=1$ to \#sub do

7: $\quad$ Pick the $\left\{(\right.$ temp2-1)+w $\}$-th of the set $S$ to be the element of the set $Q_{\text {temp1 }}$.

8: $\quad$ Record in the List $L_{\text {temp1 }}$

9: end for

10: Shuffle the set $Q_{\operatorname{temp1}}$ and record the original order in the List $L_{\text {temp1 }}$

11: end for

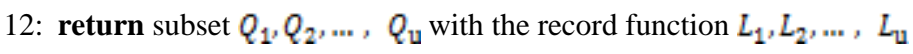




\section{ALGORITHM III: CheckResult}

Algorithm III CheckResult: When receiving the computation results, check the correctness by comparing the answers with the same calculation operation but from different assistant nodes.

Input: Several sets $S_{1 y} S_{2 y, x} S_{i}$, the function $L_{1}, L_{2 x, \ldots s} L_{i}$

Output: return error or true.

1: for the inputs from different assistant nodes but with same calculation operation do
2: if the computation results are different then
3: return error
4: end if
5: end for
6: return true

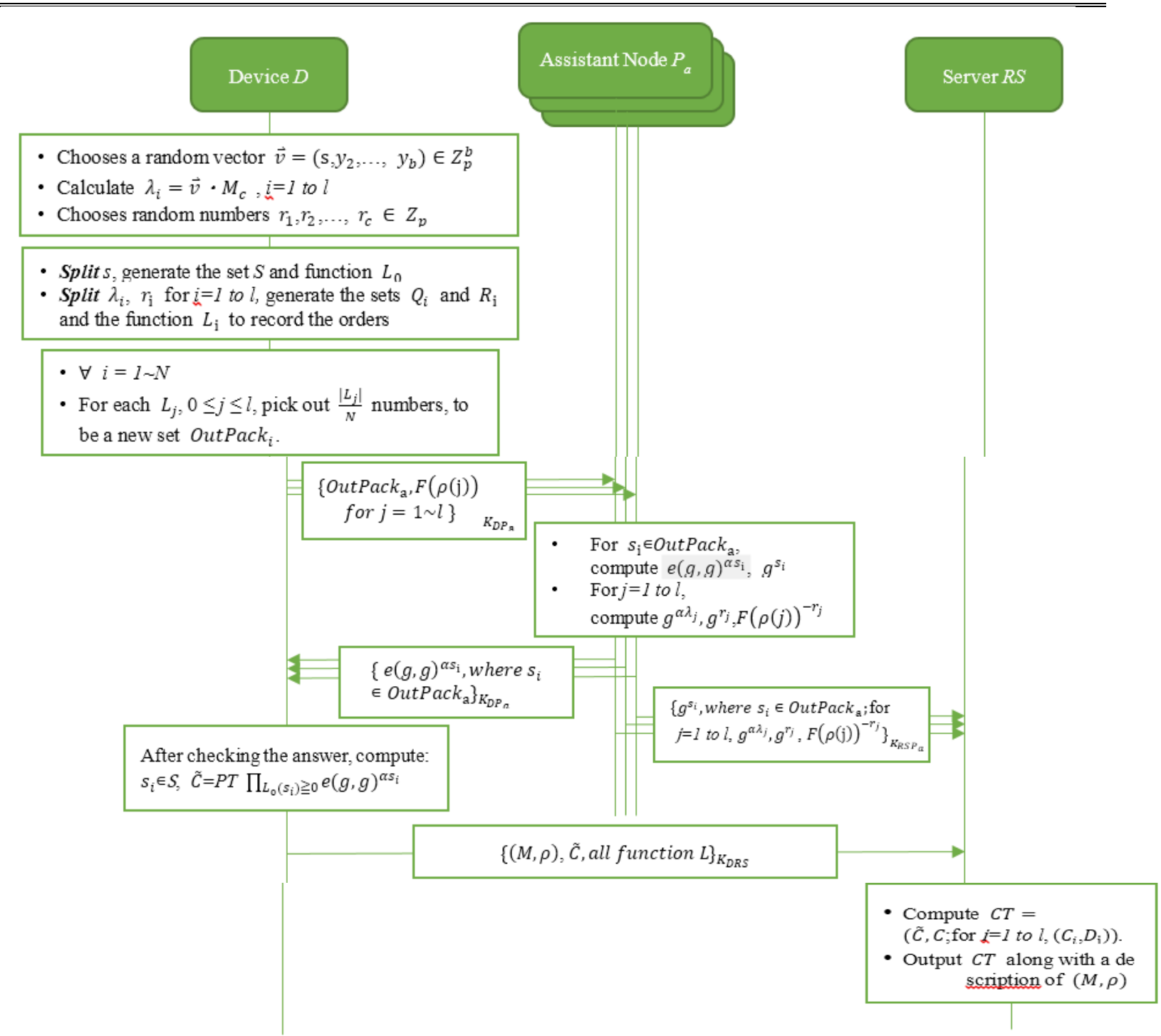

Fig 2: The diagram of the proposed protocol 


\section{Discussion}

In this section we discuss the security and the performance analyses.

\subsection{Security Analysis}

\subsubsection{Security degree}

The $\mathrm{CP}-\mathrm{ABE}$ is secure when the number of collusion nodes is less then $T$, the parameter inputted by the user. In this paper, we request that $T$ must be larger than 2 .

\subsubsection{Computational accuracy}

For example, when the user set $T \geq 2$, it means the computation would be check two times (or more) every two computation parts (with the same computation operation). In the condition of no collusion node exists, the probability of cheating by a malicious node without being detected is negligible (near to zero) (we ignore the probability of two nodes returning the same wrong answer without collusion).

\subsection{Performance Analysis}

\subsubsection{Computation cost}

Because of outsourcing the exponentiation computation to the assistant nodes, the device D has no exponentiation, but it has to compare the outsourcing computation result $|S| \times \mathrm{T}$ times and do the multiplication $|\mathrm{S}|$ times.

\subsubsection{Communication cost}

The communication times of the device $D$ is (the number of the assistant node) $\times 2+1$.

\section{Conclusions and Future Works}

This paper presented a new approach to outsource the encryptions within the IoT environment. The advantage of the new approach is that it has the ability to check the outsourcing results without heavy computational cost. The decryption part can follow the methods of $[4,7]$ to achieve both of encryption and decryption outsourcing in the CP-ABE for IoT environment.

In the future, it would be interesting to find out the best solutions of some parameters like security degree $T$, the number of split part, etc. by the simulation experiments to replace the heuristic inputs by the users.

\section{Acknowledgements}

This research is supported by Ministry of Science and Technology, Taiwan under the grants: MOST 1052221-E-415-015 and MOST 106-2221-E-415-003-MY2.

\section{References}

[1] M. Asim, M. Petkovic, and T. Ignatenko. "Attribute-based Encryption with encryption and decryption outsourcing," 12th Australian Information Security Management Conference (Perth, Australia, December 1-3, 2014), ECU Security Research Institute, pp. 1-28, 2014.

[2] J. Bethencourt, A. Sahai, and B. Waters, "Ciphertext-policy attribute-based encryption," 2007 IEEE Symposium on Security and Privacy (SP'07), IEEE Press, 2007.

https://doi.org/10.1109/SP.2007.11

[3] V. Goyal, O. Pandey, A. Sahai, and B. Waters. "Attribute-based encryption for fine-grained access control of encrypted data," In Proc. the 13th ACM conference on Computer and communications security, ACM, 2006.

https://doi.org/10.1145/1180405.1180418 
[4] M. Green, S. Hohenberger, and B. Waters. "Outsourcing the decryption of ABE ciphertexts," in Proc. the 20th USENIX conference on Security, 2011.

[5] X. Ma, J. Li, and F. Zhang, "Efficient and secure batch exponentiations outsourcing in cloud computing," 2012 4th International Conference on Intelligent Networking and Collaborative Systems (INCoS), IEEE Press, 2012.

https://doi.org/10.1109/iNCoS.2012.31

[6] L. Touati, Y. Challal, and A. Bouabdallah, "C-CP-ABE: Cooperative ciphertext policy attribute-based encryption for the Internet of Things," 2014 International Conference on Advanced Networking Distributed Systems and Applications (INDS), IEEE Press, 2014.

https://doi.org/10.1109/INDS.2014.19

[7] B. Waters, "Ciphertext-policy attribute-Based encryption: An expressive, efficient, and provably secure realization," Public Key Cryptography, vol. 6571, pp. 53-70, 2011.

https://doi.org/10.1007/978-3-642-19379-8_4 